\title{
8 Energy Efficient Routing
}

\author{
Ibrahim Korpeoglu \\ Department of Computer Engineering, Bilkent University, Ankara, Turkey
}

\subsection{Introduction}

The design of routing protocols for wireless sensor networks (WSNs) requires new approaches to be followed due to the different characteristics and applications. The primary objective to optimise WSNs is energy efficiency, and this is also the case for routing protocols for WSNs. This chapter introduces some of the routing protocols proposed in literature for WSNs. The communication needs in WSNs exhibit many differences from ad hoc networks or Internet. Besides unicast delivery of data packets from one node to another, there is also significant need for broadcasting data to a subset nodes, gathering of data from all nodes to a single or multiple locations, or dissemination of data to a set of nodes where the set is determined dynamically during dissemination. We call the mechanisms that a WSN implements to perform these types of delivery as routing protocols, data gathering protocols, or data dissemination protocols. We will use these three terms interchangeably in text to refer to those mechanisms.

In this chapter, first the differences between WSNs and ad hoc networks as far as routing problem is concerned are introduced. Ad hoc networks are the type of networks that have the most similar features with WSNs; however there are still many important differences that require new routing protocols to be designed and adapted for WSNs. Later in the chapter, new design approaches for WSN protocols are discussed and cross-layer design is emphasised. Then some wireless link layer technologies that can support realisation of WSNs and that can affect the design of routing protocols are discussed. The descriptions of some of the routing protocols proposed for WSNs available in the literature are included afterwards. The chapter ends with a summary.

\subsection{Need for New Routing Protocols}

There are already routing protocols designed for the dynamic and mobile environment of wireless ad hoc networks. But since there are differences between ad hoc networks and WSNs, we need to design new routing protocols for WSNs. The differences of WSNs from ad hoc networks, as far as routing is concerned, are 
many. Since WSNs are most of the time application specific, the classical layered architecture, where each network layer is independent from each other, is not a very efficient paradigm for WSNs. Therefore, cross-layer design, where a couple of layers designed together or layers interact with each other to use resources in a more efficient manner, can be a more favorable paradigm for WSNs. As a result of this, the routing layer used in WSNs is usually application aware (Hac 2003). In other type of networks, the routing layer cannot look inside and process the content of received packets. But in WSNs, the routing layer can look inside the received packets and can process the payload (data). This facilitates in-network processing of data in WSNs. For example, aggregation inside network is possible which reduces the amount of data transported and the number of packets transmitted. This saves energy. Moreover, since sensor nodes carry data belonging to a single application as opposed to ad hoc network nodes, which carry traffic of different types of applications at the same time, application specific policies can be applied. In WSNs, nodes can be assumed to be not mobile. But in wireless ad hoc networks, mobility is a common case for most of the scenarios. Therefore routing protocols for WSNs can be designed assuming there will be no mobility. This assumption facilitates more efficient design.

Nodes in both ad hoc networks and WSNs are expected to be powered by batteries and therefore they are expected to be energy constrained. But batteries used in ad hoc networks are usually rechargeable batteries and also can be replaced if they are no longer functional. In WSNs, however, we should be ready for the fact that batteries cannot be replaceable or rechargeable. This may not possible, for example, because of vast number of nodes or because of harsh deployment environments of sensor nodes. Therefore, the energy becomes more critical resource in WSNs than wireless ad hoc networks. We should design routing and related protocols so that the energy is used very efficiently and batteries can be functional for months or even years.

In ad hoc networks and in Internet, data packets are routed based on destination addresses. The destinations in these networks are specified by numerical addresses. IP addresses (unicast or multicast), MAC addresses, or some other addressing scheme can be used for this purpose, and routing is done accordingly. This is called node-centric routing. In WSNs the destination of query or data packets can be more suitably specified by the attributes of the data carried in the packets. Hence the routing decisions can be based on the data carried inside the packets. This is called data-centric routing. Moreover, WSN applications are usually interested from which location data arrives instead of caring from which sensor node data arrives. Therefore, sometimes it makes more sense to route packets based on the position of the destination. Hence, geo-centric (position based) or data-centric routing approaches may be more natural to take for routing than nodecentric approaches.

The traffic pattern in WSNs is also different than in ad hoc networks. In ad hoc networks, we can assume that the traffic is distributed uniformly among all pairs of nodes of an ad hoc network. In WSNs, this may not be a very realistic assumption. In WSNs, the traffic flow is more likely to be between one or more sinks (base stations) and a set of or all of sensor nodes. For example, we can conceive a 
lot of WSN applications where the majority of traffic is directed from all sensor nodes to a single sink node. This traffic flow characteristics can provide opportunity to design more efficient routing protocols for WSNs.

A lot of WSN applications require sensor nodes to be active only for a fraction of time; the sensor nodes can be asleep most of the time. Hence the duty cycle can be very low, less than 1 percent. In ad hoc networks this is not the case. A node participating in an ad hoc network is usually active most of the time until it is explicitly put into stand-by mode. The fact that most sensor nodes can be put into sleep mode provides an additional power efficient design opportunity for WSN routing protocols.

Scalability becomes a more important objective for WSNs than ad hoc networks. We expect an ad hoc network to have number of nodes in the order of $100 \mathrm{~s}$, or 1000s at most. But the number of nodes in a WSN is expected to be much more than this. For example, a WSN consisting of 1 million nodes is possible. Therefore a routing protocol designed for such a network should be very scalable.

Since nodes in a WSN are expected to be operated with irreplaceable batteries and in harsh environments, it is highly possible that some nodes will become nonfunctional at random times after they got deployed to the field. The routing protocols should be adaptive to those cases where some nodes fail and can not take part in routing anymore. Therefore we need routing solutions that are robust against node failures and resource lack.

The sensor nodes are also expected to be much more resource-limited than nodes in an ad hoc network in terms of energy, processing power, memory and link bandwidth. The routing protocols designed for WSNs should consider this fact. For example, if we have a WSN consisting of 1 million nodes, we can not afford to implement a table-driven routing protocol requiring each node to store a routing table of size 1 million entries.

New metrics are important for the performance evaluation of WSNs, which may not make sense for wireless ad hoc networks. For example, network lifetime (survivability) is a metric that is very commonly used for WSNs (although it may be used sometimes for ad hoc networks as well). It means how long it will take before a WSN that is installed into a region becomes no more functional.

\subsection{Sources of Energy Consumption}

One of the most important objectives of routing protocols for WSNs is energy efficiency. A routing protocol designed for WSNs should decrease the energy usage and try to maximise the network lifetime. Selecting always the lowest energy paths may not increase the network lifetime since the nodes on those paths will quickly deplete their energy, and WSN lifetime will be negatively affected. Therefore protocols that distribute the load and energy consumption evenly to nodes are more favorable (Chang 2000). There are sources of energy consumption in WSNs (Ye 2002), and those sources need to be attacked in order to save energy at the 
routing layer. We can list some of the sources of energy consumption related to communication as below:

- Packet transmissions. Each packet transmission causes energy consumption and energy consumed is proportional to the number of packets transmitted. Therefore a routing scheme that reduces the number of packet transmissions can save energy.

- Packet receptions. Each packet that is received by a node is processed and therefore causes energy consumption. The processing includes demodulation, copying of bytes, etc. Therefore routing protocols should try to reduce the packet receptions. A node sometimes receives not only the packets destined to itself, but also the packets destined to other nodes in the vicinity. This is called overhearing of packets. Each such packet is also received and processed at the transceiver electronics before actually dropped at the MAC layer. Therefore each such packet also causes energy waste. The number of overheard packets should be reduced.

- Idle listening: When a sensor node is in idle listening mode, not sending, or receiving data, it can still consume a substantial amount of energy. Therefore, a sensor node that is not sending or a receiving data should not stay in idle listening mode, but should go into sleep (be powered off). Depending on the communication technology, there might be several low-power modes available. Those modes should be used whenever possible.

- Packet size: Size of a packet determines how long a transmission will last. Therefore it is effective in energy consumption. We have to reduce the packet sizes if possible. This can be achieved, for example, by combining several packets into one large packet, or by compression.

- Distance: The distance between the transmitter and receiver affects how much output power is required at the transmitter to send the packets to the receiver; this in turn affects the energy consumption. Routing algorithms can select paths that use shorter distances between nodes and in this way can reduce energy consumption.

These sources of energy consumption can be attacked by node-level or network-level schemes. Each node can apply a node-level scheme independently; a network-level scheme, on the other hand, should be applied by cooperation of several nodes or all nodes in the network.

\subsection{New Approaches}

Many new approaches related to routing can be used to improve the performance of WSNs in many dimensions, including energy and bandwidth efficiency, delay, network lifetime, and adaptation to changes. These approaches include, but are not limited to, multi-path routing (Ganesan 2002), load balancing (Schurgers 2001), and cross-layer design (Shakkottai 2003) (Madan 2005) (Goldsmith 2002). In this 
section we will briefly look to some cross-layer strategies that also include the routing protocols and that improves energy efficiency.

Cross-layer design means designing the network layers and functions together or to be interdependent to each other to serve an objective in a better manner. For example, for energy efficiency purposes, MAC layer and routing layer, or physical layer and routing layer can be designed together and operated in an interdependent manner. There are various studies proposing cross-layer solutions that involve the routing layer (Madan 2005)(Van Hoesel 2004) (Chang 2000)(Conti 2004) (Madan 2005).

The physical and MAC layers can provide status information to the routing layer so that the routing protocols can adapt better to the changing conditions. Alternatively, the physical and MAC layers can be designed or optimised with the information available about the routing layer properties or information provided by the routing layer. For example, the LEACH protocol's routing layer requires a clustered topology to be formed, and each member in the cluster sends its data to the clusterhead (Heinzelman 2000). This property of the topology implied by the routing strategy can be used to design a CDMA and TDMA based MAC layer, where CDMA is used among clusters, and TDMA is used among members of a cluster. A clusterhead can easily coordinate a TDMA channel. This is indeed the approach followed by LEACH. LEACH can also utilise the interaction between routing layer and physical layers. After clusters are formed, each member node transmits its data to a clusterhead. Here, there are two options: 1) transmitting with a fixed power (no interaction between routing and physical layers) and interfering other nodes' transmissions and also consuming extra energy; 2) transmission with a power level that is just enough to reach to the clusterhead (requires interaction between routing and MAC/PHY layers). This second option is better, since it causes less energy consumption and less interference (which indirectly affects again the energy consumption).

If cross-layer design approach is not used, there is possibility that some functions can be executed at more than one layer, causing energy waste. For example, neighbor discovery can be done at the network layer by using network layer packets. But some link layers also provide a similar functionality. For example, in Bluetooth, a piconet has a master and slaves that are neighbors to the master. When the Bluetooth link layer is formed, the master knows its slaves and the slaves know their master. There is no need for neighbor discovery at the network layer of the master in this case. So, the routing layer can use the information about neighbors maintained at the link layer, if cross-layer interaction is allowed.

For cross-layer interaction, the routing layer should be able to receive information from the link layer and physical layer. Similarly, it may need to pass information to the link and physical layers. There are a number of ways of doing this. One is piggybacking the cross-layer information to the control or data packets that are already passed between those layers (Van Hoesel 2004). Another method is to design new messages just to pass cross-layer information. A third method can be to use a lightweight database which stores all the metrics and counters maintained at all layers of the protocol stack and to which each layer can access (Conti 2004). The information stored in such a database may include the RSSI (received signal 
strength indicator) value obtained from the physical layer, the transmit power level received again from the physical layer, the number of re-transmissions obtained from the MAC layer, the maximum link layer packet size defined at the MAC layer, and the identities of the neighbors obtained from the MAC or routing layer.

\subsection{Effects of Wireless Technology on Routing}

While WSN protocols were being developed, there have been also efforts to develop wireless communication standards that can support construction of WSNs. Two such efforts are IEEE 802.15.4 and Bluetooth standards. As a fairly new standard, IEEE 802.15.4 (IEEE 802.15.4 2003) (Gutierrez 2001) defines the physical and MAC layers of a wireless technology that can be used in WSNs to connect sensor nodes to each other and to the base stations. While 802.15.4 standard defines the physical and MAC layers, the ZigBee standard (ZigBee 2004), to be placed on top of 802.15.4, defines the higher layer protocols including the network layer protocols and application layer interfaces. Besides this, Bluetooth, a more mature short-range wireless technology (Bluetooth 2004), is also a candidate for being used as the underlying communication technology for WSNs. The lower layers of Bluetooth technology are defined under IEEE 802.15.1 standardisation activity and include the specification of physical and MAC layer protocols (IEEE 802.15.1 2005). The link layer technology (technology defining the physical and MAC layers) has an impact on the design of efficient routing protocols (Fig. 8.1). For example, use of Bluetooth technology for WSNs restricts the design space for possible routing algorithms. Bluetooth range is limited and therefore some routing schemes that assume arbitrary power control capability will not work. Bluetooth uses star topology and there is a limit on the number of slaves that can be connected to a master so the nodes will have degree constraint. IEEE 802.15.4, on the other hand, can support mesh, peer-to-peer and star topologies.

Bluetooth can be used as the link layer for moderate data-rate WSN applications, and can enable development of QoS based routing protocols for the network. ZigBee also provides support for real-time applications and QoS based traffic, but the data rate provided is much less compared to Bluetooth. In IEEE 802.15.4, the nodes constituting the network can be classified into two categories (reduced functional device and full functional device). This provides opportunity to design asymmetric protocols where less functionality will be given to simple devices, and sophisticated functionality will be given to more powerful devices. This also provides a natural foundation for clustering-based routing protocols.

Depending on the MAC layer properties, the routing scheme can be tailored to provide efficient transfer of information both in terms of energy and delay. Most MAC layers for WSNs are expected to be CSMA/CA or TDMA based. For example, the 802.15.4 technology uses a CSMA/CA based MAC protocol. This has a number of advantages including easy self-configuration and maintenance at the link layer, and possibility of efficient peer-to-peer communication between nodes in the network. The MAC layer affects the decisions that will be given at the rout- 
ing layer. For example, if the MAC layer is TDMA based, coordinated by a coordinator, peer-to-peer communication between any pair nodes in the range of each other may not be very efficient. In Bluetooth, for example, all communication between two slaves has to pass through their master node. Such a TDMA based scheme, however, combined with a tree-based routing protocol can provide an efficient joint MAC/routing solution (Zaruba 2001).
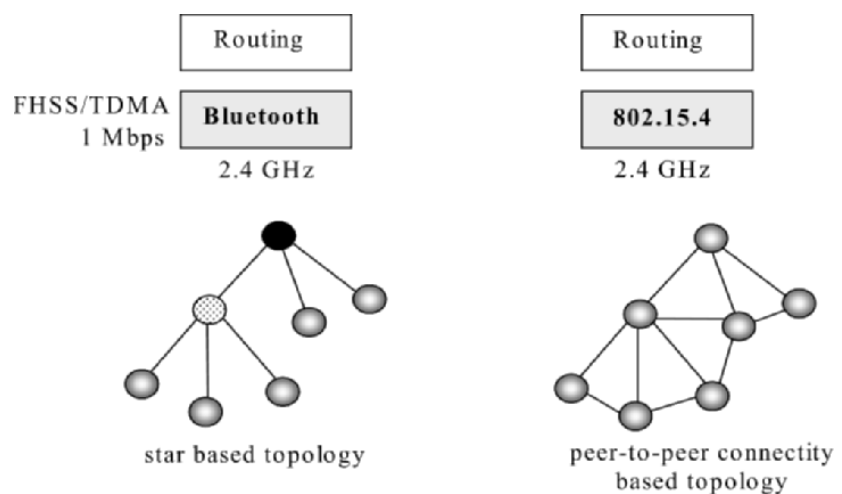

Fig. 8.1. Link layer technology and its properties affect the design of the routing protocols

Use of CSMA/CA and peer-to-peer topology at the link layer also helps matching the physical topology (defined by the range, i.e. reach ability of signals) to the logical topology (defined by the link layer connectivity). In Bluetooth, however, the physical topology can be very different than the logical topology. For example, all nodes in a Bluetooth network can be in range of each other, allowing a full mesh physical topology among nodes; but the logical topology defined by the scatternet covering all these nodes will not be a full mesh topology. It will be a topology defined by the inter-connection star-shaped small networks (piconets). What is important for the routing layer, however, is the logical topology, since it is the topology that defines who can send to whom directly.

Bluetooth networks can be built as scatternets. A scatternet is nothing but a collection of piconets inter-connected together using nodes taking roles as bridges. Therefore, most of the time, we first need to form a Bluetooth scatternet before running the routing protocol on top of it to set up the routes. The routing structures (routes or paths) can be tightly coupled with the structure of the scatternet. There are lots of scatternet formation algorithms defined in the literature that result with different shapes for the scatternets (Zaruba 2001) (Tan 2001) (Salonidis 2001) (Petrioli 2004) (Wang 2002). However, tree-based scatternets are quite common. Some scatternet formation algorithms prefer generating tree-based scatternets because of easy routing on a tree. Hence tree-based routing is a natural fit for WSNs that are based on Bluetooth technology. Tree-based routing, however, has its own deficiencies. 
Another affect of Bluetooth and 802.15.4 protocols on the routing design is visible when the addressing scheme is considered. Bluetooth link layer packets carry only a local 3-bit address, which is not enough to address all the Bluetooth nodes in a large Bluetooth network. Therefore, we need an additional addressing scheme to be used by the routing protocol designed for a large Bluetooth network. In 802.15.4, however, the link layer address range, which is also used as part of transmitted packets, is much bigger, and therefore allows these addresses to be used by the routing layer, if desired. The routing layer, however, can also use some other addressing scheme, such as IP addresses. Of course this issue is something to be concerned if only node centric communication is used in the WSN. For data centric communication it is not an important issue since forwarding is not done based on addresses.

Both technologies support sleep modes and therefore higher layers, including the routing layer, can be designed so that only a subset of all the nodes in the network can be used as active nodes and participate in routing; and the rest can be put into sleep mode or can take less responsibility. The set of active nodes can be rotated according to an algorithm and depending on the conditions.

A good approach for developing routing schemes for Bluetooth based WSNs can be considering scatternet construction and routing at the same time. Some studies follow this approach (Zhang 2005). There are also studies which focuses on a scatternet topology over which routing will be implicit and efficient. (Sain 2005) proposes a scatternet formation scheme, which produces tree-based scatternets rooted at the sink and suitable for energy efficient routing in WSNs. The constructed scatternet aims having nodes reaching to the sink over shortest paths, but also tries to balance the energy load on the nodes that consumes most power. In a tree-based topology, these are the nodes that are directly connected to the sink. Hence the formation algorithm tries to assign equal number of descendants to nodes that are directly connected to the sink. The scheme assumes there is no data aggregation applied. Therefore, the number of descendants of a node determines the energy load of the node. The scheme also considers the limited range of Bluetooth nodes and does not make an assumption requiring every node to be reachable from every other node. The piconet properties of Bluetooth are considered and the degrees of nodes are constrained.

\subsection{Routing Classification}

The design space for routing algorithms for WSNs is quite large and we can classify the routing algorithms for WSNs in many different ways. Table x.1 shows some possible classification criteria for WSN routing protocols together with some example protocols meeting the criteria. Those protocols, which are given as examples, are described in the next section.

Classify the protocols based on the way the destinations are specified. Nodecentric, location-based (geo-centric), or data-centric specification of destinations is possible in WSNs. Most ad hoc network routing protocols are node-centric proto- 
cols where destinations are specified based on the numerical addresses (or identifiers) of nodes. In WSNs, node-centric communication is not a commonly expected communication type (Niculescu 2005). Therefore, routing protocols designed for WSNs are more data-centric or geo-centric. There are routing protocols for WSNs, like LEACH (Heinzelman 2000) and PEDAP (Tan 2003), where there is a single destination, which is the sink node. Those protocols can be considered as a special case of node-centric communication. But a significant number of routing protocols designed for WSNs are data-centric. Directed Diffusion (Intanagonwiwat 2000), ACQUIRE (Sadagopan 2003), Epidemic Algorithms (Akdere 2006), Cougar (Yao 2002) are example protocols that follow data-centric communication paradigm. Specification of a destination based on geographical coordinates (location) or geographical attributes is also common. GPSR (Karp 2000), GAF (Xu 2001), and GEAR (Yu 2001) are example protocols for location-based routing.

Classify the protocols based on whether they are reactive or proactive. A proactive protocol sets up routing paths and states before there is a demand for routing traffic. Paths are maintained even there is no traffic flow at that time. LEACH and PEDAP, for example, are proactive protocols. SPIN (Heinzelman 1999), on the other hand, is a reactive protocol. The routing protocol actions are triggered when there is data to be sent and disseminated to other nodes. Directed Diffusion and ACQUIRE can be considered as reactive protocols, since paths are setup on demand when queries are initiated. Otherwise, no path setup is performed.

Classify the protocols based on whether they are destination-initiated or source-initiated. A source-initiated protocol sets up the routing paths upon the demand of the source node, and starting from the source node. A destinationinitiated protocol, on the other hand, initiates path setup from a destination node. We can consider LEACH as a destination initiated protocol since the sink triggers the setup process. SPIN, on the other hand is a source-initiated protocol. The source advertises the data when available and initiates the data delivery. Pushbased Epidemic Algorithms are also source-initiated. Directed Diffusion, on the other hand, is a destination (sink) initiated protocol.

Classify protocols based on sensor network architecture. Routing protocols depend on the architecture of the WSNs and vice versa. Some WSNs consist of homogenous nodes, whereas some consist of heterogeneous nodes. A hierarchical routing protocol is a natural approach to take for heterogeneous networks where some of the nodes are more powerful than the other ones. Therefore we can classify the protocols based on whether they are operating on a flat topology or on a hierarchical topology. The hierarchy does not always depend on the power of nodes. Hierarchy can be defined also in a physically homogenous network by assigning different responsibilities to nodes. The responsibility assignment can change dynamically during the lifetime of the network. The responsibility to a node can be given as a clusterhead, leader, gateway, or as an ordinary node. LEACH and TEEN (Manjeshwar 2001) are examples for hierarchical protocols. PEDAP and PEGASIS (Lindsey 2002), on the other hand, are not hierarchical; each node has equal responsibility. Directed diffusion is also a non-hierarchical protocol. 
Table 8.1. Some example routing protocols that have those features

\begin{tabular}{|l|l|}
\hline \multicolumn{1}{|c|}{ Property } & \multicolumn{1}{c|}{ Example Protocol(s) } \\
\hline \hline Node-centric & LEACH, PEDAP, PEGASIS \\
\hline Data-centric & Directed Diffusion, ACQUIRE, SPIN \\
\hline Geo-centric & GEAR \\
\hline Proactive & LEACH, PEDAP \\
\hline Reactive/On-demand & SPIN, Directed Diffusion, Epidemic Algorithms \\
\hline Dst-initiated & Directed Diffusion, LEACH \\
\hline Src-initiated & SPIN, Epidemic Algorithms \\
\hline Flat topology & Directed Diffusion, SPIN \\
\hline Hierarchical topology & LEACH \\
\hline Regular Structure & PEDAP \\
\hline Irregular structure & Directed Diffusion \\
\hline No structure & Epidemic Algorithms, SPIN \\
\hline Centralised/ Distributed & PEDAP/Directed Diffusion, LEACH \\
\hline Multipath-based & EAR, Directed Diffusion \\
\hline Singlepath-based & PEGASIS, LEACH \\
\hline
\end{tabular}

Classify the protocols based on whether they use a regular routing structure. Some protocols build a regular structure over which routing will take place prior to actually routing the packets. LEACH, PEDAP, PEGAS are examples of such protocols. Some other protocols do not build a regular structure for routing; for example, Directed Diffusion. On the other hand, some protocols do not have any structure at all: they do not maintain state information. For example, SPIN disseminates data just based on local interactions and do not establish paths from sources to destinations. Similarly, Epidemic Algorithms, Rumor Routing (Braginsky 2002), and Gossiping (Hedetniemi 1988) protocols can be considered as having no structure, i.e. maintaining no end-to-end paths. Classify the routing protocols based on protocol operation. Multipath routing and QoS based routing are example categories that are based on the operation of the protocol. The QoS based protocols operate considering the QoS that can be supported by the paths. Multipath routing protocols use more than one path to route the flows and packets. For example, the Energy Aware Routing (EAR) protocol proposed in (Shah 2002) uses multiple paths to route packets towards the sink. Although this causes some sub-optimal paths to be used, it distributes the load more evenly to sensor nodes and prolongs the network lifetime. Classify the protocols based on whether they are centralised or distributed. Distributed computation of routing paths and states is preferable for WSNs, which are highly dynamic and self-organising. But computing the routing paths at a central location has also some advantages like providing optimal paths, easy implementation, etc. Therefore, we can design routing protocols so that they compute the paths in a distributed manner or in a central location. For example, PEDAP computes the paths at a central location and then informs the sensor nodes to follow the computed structure. Direction Diffusion, SPIN, and LEACH, on the other hand, are example protocols that compute the paths in a distributed fashion. 


\subsection{Routing Schemes}

Table 8.2. There are different applications for WSNs. Each application has its own traffic characteristics and this affects the selection of the most suitable routing protocol

\begin{tabular}{|l|l|l|}
\hline \multicolumn{1}{|c|}{ Application } & \multicolumn{1}{|c|}{ Traffic Properties } & \multicolumn{1}{|c|}{$\begin{array}{c}\text { Suitable Routing } \\
\text { Protocols }\end{array}$} \\
\hline \hline $\begin{array}{l}\text { Environmental } \\
\text { Monitoring }-1\end{array}$ & $\begin{array}{l}\text { All nodes send data periodically } \\
\text { to sink }\end{array}$ & $\begin{array}{l}\text { LEACH, PEGASIS, } \\
\text { PEDAP }\end{array}$ \\
\hline $\begin{array}{l}\text { Environmental } \\
\text { Monitoring - } 2\end{array}$ & $\begin{array}{l}\text { Nodes send data when an event } \\
\text { occurs }\end{array}$ & $\begin{array}{l}\text { Directed Diffusion, } \\
\text { Epidemic Algorithms, } \\
\text { SPIN }\end{array}$ \\
\hline $\begin{array}{l}\text { Sink issues sin- } \\
\text { gle-shot queries } \\
\text { and some nodes } \\
\text { reply }\end{array}$ & $\begin{array}{l}\text { A query has to be disseminated; } \\
\text { data has to be transported to the } \\
\text { sink from nodes that can answer }\end{array}$ & $\begin{array}{l}\text { Cougar, Acquire, } \\
\text { GEAR }\end{array}$ \\
\hline $\begin{array}{l}\text { Sink issues long- } \\
\text { running queries }\end{array}$ & $\begin{array}{l}\text { A query has to be disseminated. } \\
\text { Data has to be transported to the } \\
\text { sink from nodes that can an- } \\
\text { swer; this happens for a long } \\
\text { duration. }\end{array}$ & $\begin{array}{l}\text { Flooding, Directed } \\
\text { Diffusion, LEACH, } \\
\text { PEGASIS, PEDAP }\end{array}$ \\
\hline
\end{tabular}

There are plenty of routing algorithms proposed in the literature for WSNs. The choice of which routing protocol to use for a WSN depends on the application to run and the traffic properties of the application. Table x.2 shows the description of some application types, their traffic properties and some suitable WNS routing protocols. In this section, some widely known routing protocols will be described briefly.

\subsubsection{LEACH}

LEACH is a clustering-based hierarchical routing protocol designed for WSNs. The protocol requires a set of sensor nodes to be elected as clusterheads according to a randomised algorithm. The set of nodes that are clusterheads are rotated during the lifetime of the network. Each rotation is called a round. In this way, the energy load is more evenly distributed to sensor nodes, since being a clusterhead is much more energy consuming than being a member node (Fig. 8.2). LEACH is designed for WSN applications where there is a single sink node and all sensor nodes are required to sense the environment periodically and send the data to the sink. Hence the data communication is directed towards the sink; the only destination. The protocol is not suitable for applications that require some sensor nodes to be destinations as well. The protocol, in each round, first elects a percentage (5\%) of sensor nodes as clusterheads. Each sensor node is assigned to a clusterhead. Then, at each sensing period, all sensor nodes send their data to their clusterheads. 
A clusterhead aggregates the data received from member nodes into a single packet and transmits it to the base station (sink). Hence a data packet originating at a sensor node reaches to the sink after two hops. This provides quite fast delivery of data to the sink in an energy efficient manner. But being able to reach to the base station in two hops requires the nodes to have power control capability so that they can adjust their power level to reach to arbitrary distances. If they cannot do this, then the coverage becomes limited and LEACH cannot be used for WSNs deployed over large areas in this case. The percentage of clusterheads does not have to be fixed to $5 \%$. It can be increased or decreased. If it is increased, it means more clusterheads will be on the field and therefore the average distance that sensor nodes will transmit to reach clusterheads will be reduced. On the other hand, more packets will be transmitted from the clusterhead to the sink. The clusterhead selection algorithm promotes uniform distribution of clusterheads to the field. Some of these clusterheads, which are far away from the base station, will spend more energy than the other ones. The clustering based architecture of LEACH, the property that each clusterhead sends its data directly to the base station, and rotating the clusterheads provide an important advantage which is distributing the load more evenly to sensor nodes. In multi-path based routing protocols, which may be operating on a tree-like topology, we have the problem of uneven distribution of load to sensor nodes that are close to the root (sink), since all traffic has to go through those nodes. In LEACH, however, this is not a problem since the clusterheads are distributed evenly to the sensor field and they are rotated at every round.

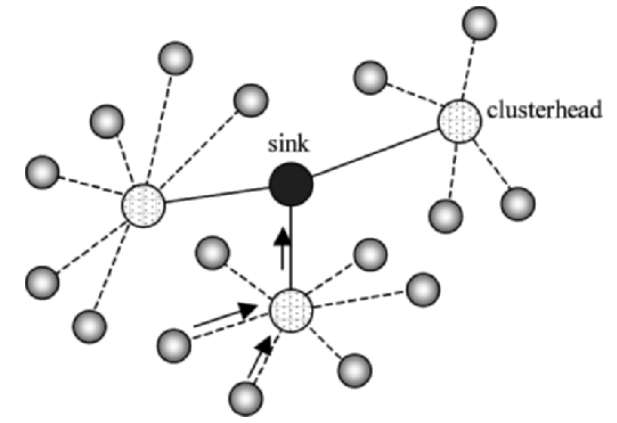

Fig. 8.2. LEACH routes packets in two hops. Clusters are formed, and each node sends its data packet to its clusterhead. Each clusterhead aggregates the data received from the cluster members into a single packet and sends it to the sink.

\subsubsection{PEDAP}

PEDAP (Tan 2003) is another routing scheme that is suitable for WSN applications that require periodic sensing of environment by each sensor node and sending of the sensed information to a sink node. It is assumed that data aggregation at 
intermediate nodes is possible for the applications. The data flow is again directed from all sensor nodes to a single point, which is the sink node. Again, power control capability at each node is needed for PEDAP to operate efficiently. PEDAP is based on the idea that at each round of communication (during which data from all sensor nodes is delivered to the sink once) the network should consume minimum amount of energy and the energy load should be balanced among the nodes so that a longer lifetime can be achieved. To spend less energy per round, a minimum spanning tree (MST) spanning all the sensor nodes and rooted at the base station is constructed, and routing is performed over the edges of this tree (Fig. 8.3). Accordingly, each node will receive data from one or more sensor nodes (children in MST), aggregate the received data, and then will send the aggregated data to the next sensor node (parent in MST) on the way towards the sink. The cost of each link (edge) is the energy consumed for sending a packet over that link. This energy depends on the size of the packet, the distance between sender and receiver, and the transceiver electronics energy consumption constants (Heinzelman 2000). Hence, routing over MST implies minimum total energy consumption per round.

If the distance between a node $i$ and $j$ is $d_{i j}$, then the cost of the edge connecting those nodes depending on the packet size $k$ is computed as follows:

$$
C_{i j}(k)=2 E_{\text {elec }} k+E_{\text {amp }} k d_{i j}^{2}
$$

Similarly, the cost of an edge connecting a sensor node $i$ to the sink (which is mains powered) is computed as follows (again for a packet of size $k$ ):

$$
C_{i}^{\prime}(k)=E_{\text {elec }} k+E_{\text {amp }} k d_{i b}^{2}
$$

Here, $E_{\text {elec }}$ and $E_{\text {amp }}$ are radio transceiver dependent constants. This cost model is based on the radio model proposed by (Heinzelman 2000).

PEDAP also tries to distribute the energy load evenly to sensor nodes by increasing the cost of edges connected to nodes that have very little energy left. For that, it modifies the edge cost model by dividing it with the remaining energy of the node.

This implies that, the cost of an edge connected to the node increases, while the remaining energy of the node decreases. Hence, a node that has very little energy left will have a very high cost for its edges and therefore those edges will be excluded from the minimum spanning tree and will not be used for routing in the next phase. In this way, traffic load on this node will be reduced. PEDAP reconstructs the MST periodically (at each phase), which means the remaining energy levels of nodes are checked and taken into account periodically.

The route computation in PEDAP is done at a central location and the position of sensor nodes are assumed to be known. Performing route computation in a centralised manner has the advantage of obtaining better routes and preventing routing loops. But it has also some drawbacks. Maintenance of routes is more difficult when nodes fail or deplete their energy or when links break. 


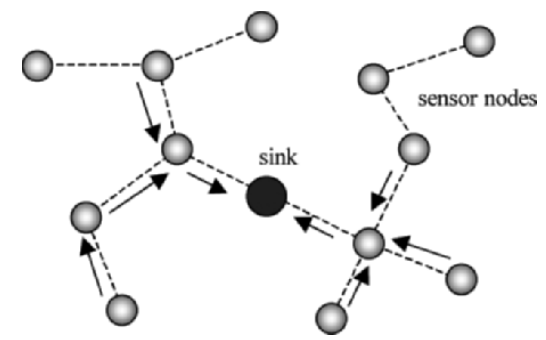

Fig. 8.3. A sensor network, where routing paths are established by the PEDAP protocol PEDAP builds an MST spanning the sensor nodes and rooted at the sink. The packets are routing over the edges of the MST

\subsubsection{PEGASIS}

PEGASIS (Lindsey 2002) is a routing scheme that is based on forming a chain passing through all sensor nodes, and packets are routed over this chain (Fig. 8.4). The chain is formed before data gathering process starts. Each node on the path aggregates the received data with its own data and sends the aggregated data to the next node on the chain. One node on the chain is selected as the node that will finally forward the aggregated data to the base station. This node partitions the chain into two. The data is collected at this node starting from each end of the chain. The responsibility of sending the aggregated data to the sink is rotated among the sensor nodes, and in this way energy load is more evenly distributed.

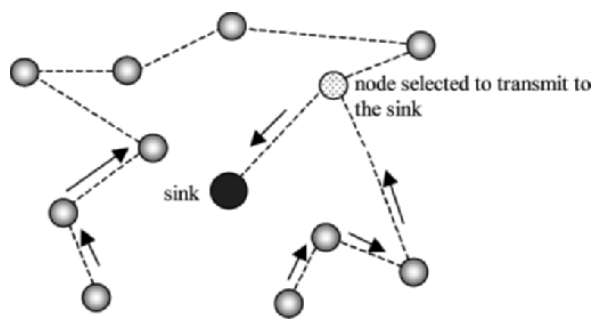

Fig. 8. 4. The PEDAP protocol establishes a chain passing through all sensor nodes. A node on the chain is selected to transmit the data to the base station. Data is transported on the chain after getting aggregated at each node

A greedy algorithm can form the chain. Starting at a node, each node selects a nearest neighbor to be the next node on the chain. In this way, the transmission energy cost over the chain is reduced, since the distances between adjacent nodes are reduced. This greedy approach, however, does not give an optimal solution. The optimal solution is the solution of the TSP (traveling salesman problem) problem. The disadvantage of PEGASIS is the long delays for packets sent by the 
nodes that are close to the ends of the chain. The distance of these nodes to the node that is transmitting to the sink is $\mathrm{O}(n)$ where $n$ is the number of nodes in the network. PEGASIS can work in environments where range is limited, provided that there are neighbors of each node that are in the range. But this does not guarantee forming a chain. PEGASIS is more scalable compared to LEACH in terms of the area of the region is concerned, since it does not have two-hops limitation.

\subsubsection{Directed Diffusion}

Directed Diffusion is a data-centric routing protocol (Intanagonwiwat 2000) designed for WSNs. The routing and path setup decisions are made according to the data that packets deliver. Directed diffusion is developed with the aim of being a robust, scalable, and energy efficient protocol, all of which are very important objectives for the design of protocols for WSNs. In data-centric routing, the destinations are identified with the content of the packets. The content is organised in a format that consists of sequence of attribute-value pairs. A setting of those attributes specifies the destinations that will process the packet. Directed diffusion is a distributed protocol and uses local interactions to setup paths and route packets. There is no central coordination required. It is also scalable since the state information stored at intermediate nodes is independent of the number of nodes in the network. It can work for both mono-sink and multiple-sink scenarios. In Directed Diffusion, the sinks via messages called interests activate sensor nodes. A sink that is interested in obtaining information about a region in the sensor field builds up an interest message and disseminates the message through the network. An interest message consists of attribute-value pairs. Those attributes specify what kind of information the sink would like to receive about the network. The attributes may also include the coordinates of the region from where data will collected, the rate of data collection, and the duration of the data gathering process. The interest message can also be considered as a query message or a task description message.

The dissemination of interest is realised as follows. The sink node, that originates the interest, broadcasts the interest to its neighboring sensor nodes. Each sensor node receiving an interest creates an entry in its local table for the interest and creates gradients (pointers to the next node) in the entry pointing to the nodes from which the interest is received. These gradients will be used during data delivery to forward the data packets to the next nodes towards the sink. After gradients are setup at the node, the node also broadcasts the interest to its neighbors. This process continues and the interest gets diffused through the network. Each sensor node receiving the interest checks the attributes of the interest, and if required, processes the interest. For example, if the node is inside the region specified by the target coordinates stored in the interest, then the node activates its sensors and becomes ready to send data towards the sink. When the interest is disseminated in the network, some sensor nodes that decide to process the interest will be the sources of data (and events) to be sent to the sink. When a source sensor node would like to send data to the sink, it sends the data packet to the directions (neighbors) shown by the gradients stored in the corresponding interest en- 
try. Each intermediate node that is receiving a data packet checks its interest table and finds the matching interest entry. The matching interest entry will have gradients showing the next hops (neighbors) the data packet has to be forwarded. The node then forwards the packet to those neighbors. In this way, the data packet is routed back to the sink node, possibly over multiple paths.

It is not desirable to receive the same information over multiple paths. It causes energy waste. Therefore, Directed Diffusion has a path-reinforcement mechanism through which a single path among several paths is selected and only that one is used for further information sent from the source to the sink. This is achieved by reinforcing the neighbors along the selected path, starting from the sink node. Reinforcement is done by sending a higher rate interest message starting at the sink node and along the selected path towards the source node. The gradients corresponding to the links of this selected path will be involved in activity. Each gradient setup in Directed Diffusion protocol has a timer associated with it. If no packet is sent over that gradient for the time period for which a timer has been setup, the gradient is deleted. In this way, the gradients corresponding to the paths that are undesired will be deleted with time and only one path will be used to deliver packets from source to the sink.

\subsubsection{SPIN}

SPIN (Heinzelman 1999) is also a data-centric data dissemination scheme that is distributed, negotiation-based, and resource-aware. It can be considered as reactive (on-demand) protocol where data delivery is initiated by the source node. It is more suitable for applications where data will be available at unpredictable times. It is an energy and bandwidth efficient protocol. SPIN is a negotiation-based protocol. Neighboring sensor nodes negotiate with each other for transfer of information. For this, meta-data based approach is used to describe the data that is to be disseminated to other nodes. It is assumed that the size of the meta-data will be much smaller compared to the size of the corresponding data for most applications. Therefore, meta-data is exchanged between neighbors before the actual data is delivered. A node that has new data first advertises the availability of the data to its neighbor (by using an ADV - advertisement - message). Any neighbor that does not have the data and is interested in receiving the data, requests the data explicitly (by using a REQ - data request - message) upon receiving the advertisement. Then the data is transmitted (by using a DATA - payload - message). If there is no neighbor that would like to receive the data, then the data is not transmitted. Any node receiving a new data from a neighbor applies the same procedure: it advertises the new data to its neighbors and the interested neighbors request the data. This process continues as long as there are nodes that are interested in receiving the data that is advertised by some neighbor. In this way, transmission of redundant information is eliminated. SPIN is also resource-aware and resourceadaptive. It requires each node to monitor its own resources, such as remaining battery energy, traffic load, etc., and adjust its behavior and activities accordingly. 
SPIN aims to solve the following problems that can be seen in flooding-based data dissemination schemes: 1) implosion; 2) overlap; and 3) resource blindness. Implosion means that a node receives the same data packet more than once, possibly through different paths. Overlap means that several data items that is received by a node correspond or include the same information. For example, two sensor nodes may sense overlapping regions and therefore produce data packets that include overlapping information. Resource blindness is acting of sensor nodes without caring about the resources consumed and the level of available resources. SPIN attacks the first two problems by use of meta-data and negotiations. It attacks the third problem by being resource-aware. It adapts the activities of a node based on the level of resources the node has.

\subsubsection{Epidemic Algorithms}

Epidemic Algorithms are based on the theory of epidemics that is about the models for spread of diseases. Epidemic Algorithms follow these natural models to disseminate information in networks and distributed environments (Demers 1987). These models are using interaction between neighbors to disseminate information, as in SPIN. The interaction can be pull-based or push-based. In pull-based interaction and delivery, a node requests information from a neighbor and gets the information if it is available at the neighbor. In push-based interaction, a node that has some new information sends it to a selected neighbor. In this way the information is delivered to the neighbor and the neighbor can execute the same steps to disseminate the information further. The information that a node has can be cached in the node for a while even though it is sent to a neighbor. There are different policies defining when to delete an information message from the cache. This approach for delivering information in a distributed environment can also be used in WSNs (Akdere 2006). Sensor nodes can push data to their neighbors by local interactions. Similarly a sink node, or another sensor node can pull data from neighbors by local exchange of messages. An important property of Epidemic Algorithms is that they are robust to changes in the network. Links can break, nodes can move or fail, but the algorithms still work and deliver data. Epidemic Algorithms are realised by distributed protocols requiring local interactions. There is no need for a central control. There is also no path setup needed to deliver messages between nodes. This is something different than Directed Diffusion. Directed Diffusion is also a distributed protocol and is robust, but it first establishes paths between sources and destinations via gradients set up at intermediate nodes.

\subsubsection{ACQUIRE}

ACQUIRE is a data gathering scheme that is more suitable for WSN database applications (Sadagopan 2003). In ACQUIRE, a query is resolved while it is being disseminated. In other words, a query is processed inside the network, as opposed to being processed at a central location after all data is available at the central lo- 
cation. ACQUIRE aims improving energy efficiency by not sending a query to all nodes and by not requiring all sensor data to be collected first at the center before processing. Therefore it is more efficient than flooding based approaches, and can be used efficiently for one-shot queries for which flooding the whole network would cause too much overhead. It is also suitable for complex queries that contain several interests and variables, and that can be progressively resolved at intermediate nodes. In this way, the amount of data packets (and their sizes) that should be transported to the sink is reduced.

ACQUIRE works as follows. The sink injects an active query, which can be a one-shot or complex query, into the network. Any node receiving the query tries to answer (resolve) the query as much as it can. This is achieved by the cached information at the node that is received from $d$-hop neighbors of the node. If the cached information is out-of-date (has been received long time ago), the node can initiate a data request from its $d$-hop neighbors upon receiving the query. In this way, the node will have information about its local neighborhood and this information is used to resolve the query as much as possible. If the query is completely resolved, we are done and the query is propagated back to the sink.

If the query is not completely resolved, it is sent to another sensor node in the network which will further process the query. That node can be determined in a random manner, or by an intelligent decision. That node receiving the query will execute a similar procedure to process the query as much as possible. If the query is still not completely resolved, it is sent to another node. In this way, the query is processed step-by-step inside the network. Hopefully, most of the time, the query will be completely resolved without needing to travel along all the sensor nodes.

\subsubsection{GEAR}

GEAR - Geographical and Energy Aware Routing - (Yu 2001) is a routing protocol that is location-based and energy-aware. It makes routing decisions based on the coordinates of a destination region that packets are destined to. Therefore, a packet has to include information about the region. The region can be specified as a rectangle and its coordinates can be included in the packet. Assume such a packet is to be delivered to all nodes in the rectangular region located somewhere in the field where WSN is deployed. GEAR performs the delivery in two steps. 1) First it delivers the packet from the source node (which can be the base station) to a node in that region. For this, it uses location-based and energy-aware routing. 2) Then, when the packet arrives to a node inside that region, that node delivers the packet to all nodes in the region by Recursive Geographic Routing. If this may not work for some scenarios, then restricted flooding can be used as well.

Forwarding of a packet towards the destination region is achieved by a combination of geographical and energy-aware route selection techniques. GEAR assumes that each node knows its neighbors, their positions, and their remaining energy levels. This can be achieved by infrequent message exchanges between neighbors. Using these values at a node, GEAR computes a cost value for each neighbor of the node. Using these costs, and the costs of sending packets from the 
node to each neighbor, GEAR selects a neighbor to forward the packet next. As a result of this algorithm, when all neighbor nodes have equal level of energy, for example, the protocol selects the neighbor that is closest in distance to the destination region. On the other hand, when all neighbors have equal distance to the destination region, then the protocol selects the neighbor that has the largest remaining energy. In this way, GEAR tries to both balance energy load on neighbors and route the packets to the destination over shorter distances.

When a packet arrives to a destination region, it is delivered to all nodes in the region using Recursive Geographic Routing. For this, the destination region is first divided into four sub-regions and a copy of the packet is sent to each subregion using location-based routing. Then, when a copy of the packet arrives to a sub-region, the same procedure is applied in that sub-region as well: it is further divided into four other sub-regions and a copy of packet is sent to each sub-region. This continues until a sub-region has just one node. As a result of this Recursive Geographic Routing, all nodes in the destination region can receive the packet sent from the source.

GEAR enables efficient routing of a packet to a destination region. This is usually required, for example, when a base station issues a query and the query has to be forwarded to all nodes in a destination region. GEAR provides an energyefficient and location-based routing protocol for delivering the query. The same protocol can be used, however, for sending the data packets from those nodes to the sink back. All sensor nodes can know the location information of the sink, and the data packets generated by these sensor nodes can be targeted to that location. That delivery can also be achieved by the location-based and energy-aware forwarding mechanism of GEAR.

\subsection{Summary}

This chapter describes the routing problem in WSNs. The routing problem is also identified as data dissemination or data gathering problem in WSNs. Although there are some differences between data dissemination, data gathering, and data routing, what all aim is efficient delivery of data packets from one or more sources to one or more destinations. Therefore, here in this chapter, routing, data dissemination, and data gathering are considered as the same problem in WSNs, and they are unified under a single topic: routing and data dissemination protocols for WSNs.

WSNs are sometimes considered as a sub-class of ad hoc networks. But there are actually very important differences. WSNs consist of unmanned nodes which are powered by irreplaceable batteries. Therefore energy is the most important resource. The number of nodes in a WSN can be much larger than the number of nodes in an ad hoc network; therefore scalability of protocols is very important. The traffic is usually directed from or to a base station in WSNs. This provides opportunity to design special routing protocols tailored for this property. WSNs are also application-specific networks and therefore it is possible to design routing 
protocols that are application-aware and application-specific. There are a number of wireless technologies that can be used as the link layer technology in WSNs. Bluetooth and IEEE 802.15.4 are two such technologies. The properties of the link layer technology affect the design of routing protocols. The topologies that can be generated by the link layer, the MAC protocol, the bit-rate, data range, and whether it is possible to adjust the transmission power are all important factors that can affect the design and optimisation of the routing protocols for WSNs.

Several routing protocols specifically designed for WSNs are introduced in this chapter. Those routing protocols can be classified in many different ways: 1) based on whether they are node-centric, geo-centric, or data-centric; 2) based on whether they operate on a flat topology or on a hierarchical topology; 3) based on the whether they are reactive or proactive; etc. Most routing protocols designed for WSNs follow a data-centric approach where the destinations are specified not by numerical addresses, but by values of the attributes of the data included in the packets. Data-centric routing is a new approach that is proposed for WSNs. Since WSNs are application-specific, the design space for routing algorithms for WSNs is very large. Each WSN and application may require its own routing protocol to be designed; but still there are some common approaches and mechanisms that can be followed by routing protocols designed for many different applications. The chapter therefore has provided the descriptions of some of the widely known routing protocols developed for WSNs. These protocols encompass many of the important design approaches and techniques for routing in WSNs.

\subsection{References}

Akdere M, Bilgin C, Gerdaneri O, Korpeoglu I, Ulusoy O, and Cetintemel U (2006) A comparison of epidemic algorithms in WSNs. Computer Communications (to appear)

Akkaya K and Younis M (2005) A survey on routing protocols for WSNs. Ad hoc networks 3:325-349

Al-Karaki JN and Kamal AE (2004) Routing techniques in WSNs: a survey, IEEE Wireless Communications 11:6-28

Bluetooth Special Interest Group (2004) Bluetooth core specifications. Available at http://bluetooth.com/Bluetooth/Learn/Technology/Specifications/

Braginsky D and Estrin D (2002) Rumor routing algorithm for sensor Networks. In: Proc. of $1^{\text {st }}$ Workshop on Sensor Networks and Applications, Atlanta

Chang JH and Tassiulas L (200) Energy conserving routing in wireless ad-hoc networks, In: Proc. of IEEE INFOCOM. pp 22-31

C. $-\mathrm{K}$. Toh (2001) Maximum battery life routing to support ubiquitous mobile computing in wireless ad hoc networks. IEEE Communications Magazine 39:138-147

Conti M, Maselli G, Turi G, and Giordano S (2004) Cross-Layering in mobile ad hoc network design. IEEE Computer 37:48-51

Demers A, Greene D, Hauser C, Irish W, Larson J, Shenker S, Sturgins H, Swinchart D, and Terry D (1987) Epidemic algorithms for replicated database maintenance. In: The Proc. of the ACM Symp. on principles of distributed computing. pp 1-12 
Ganesan D, Govindan R, Shenker S, and Estrin D (2002) Highly resilient, energy efficient multipath routing in WSNs. Mobile Computing and Communications Review 5:11-25

Ganesan D, Krishnamachari B, Woo A, Culler D, Estrin D, and Wicker S (2002) An emprical study of epidemic algorithms in large-scale multihop wireless networks. (Technical report UCLA/CSD-TR-02-0013 by the Department of Computer Science of UCLA)

Goldsmith AJ and Wicker WB (2002) Design challenges for energy-constrained ad hoc wireless networks. IEEE Wireless Communications 9:8-27

Goussevskaia O, Machado MV, Mini RAF, Loureiro AAF, Mateus GR, and Nogueira JM (2005) Data dissemination on the energy map. IEEE Communications 43:134-143

Gutierrez JA, Naeve M, Callaway E, Bourgeois M, Mitter V, and Heile B (2001) IEEE 802.15.4: a developing standard for low-power low-cost wireless personal area networks. IEEE Network 15:12-19

Hac A (2003) WSNs designs. Wiley

Hedetniemi S and Liestman A (1988) A survey of gossiping and broadcasting in communication networks. IEEE Network 18:319-349

Heinzelman WR, Chandrakasan A, and Balakrishnan H (2000) Energy-efficient communication protocol for wireless microsensor networks, In: Proc. of the Hawaii Int. Conf. on System Sciences

Heinzelman WR, Kulik J, and Balakrishnan H (1999) Adaptive protocols for information dissemination in WSNs. In: Proc. of ACM/IEEE, Int. Conf. on mobile computing and networking. Seattle, pp 174-185

IEEE 802.15.1 (2005) IEEE 802.15.1 Standard Specification. Available from

http://standards.ieee.org/getieee802/download/802.15.1-2005.pdf

$\begin{array}{lllll}\text { IEEE } & \text { 802.15.4 } & (2003) & \text { Standard } & \text { Specification. Available from }\end{array}$ http://www.ieee802.org/15/pub/TG4.html

Intanagonwiwat C, Govindan R, and Estrin D (2000) Directed diffusion: A scalable and robust communication paradigm for sensor networks, In: Proc. of the ACM/IEEE Int. Conf. on mobile computing and networking. Boston, pp 56-67

Karp B and Kung HT (2000) GPSR: Greedy perimeter stateless routing for wireless networks. In: Proc. of the $6^{\text {th }}$ Annual Int. Conf. on mobile computing and networking. pp $243-254$

Lindsey S and Raghavendra CS (2002) PEGASIS: power-efficient gathering in sensor information systems, In: Proc. of IEEE Aerospace Conf.. pp 1125-1130

Madan R, Chui S, Lall S, and Goldsmith A (2005) Cross-Layer design for lifetime maximisation in interference-limited WSNs. In: Proc. of IEEE INFOCOM. pp 1964-

Manjeshivar A and Agrawal DP (2001) TEEN: A routing protocol for enhanced efficiency in WSNs. In: Proc. of the Int. parallel and distributed processing Symp. workshops

Niculescu D (2005) Communication paradigms for sensor networks. IEEE Communication Magazine 43:116-122

Petrioli C, Basagni S, and Chlamtac I (2004) BlueMesh: degree-constrained multi-hop scatternet formation for Bluetooth networks. Mobile Networks and Applications 9:33-47

Sadagopan N, Krishnamachari B, and Hemly A (2003) The ACQUIRE mechanism for efficient querying in sensor networks, In: Proc. of the IEEE Int. Workshop on Sensor Network Protocols and Applications. pp 149-155

Saginbekov S and Korpeoglu I (2005) An energy efficient scatternet formation algorithm for Bluetooth based sensor networks. In: Proc. of the European Workshop on WSNs. Istanbul 
Salonidis T, Bhagwat P, Tassiulas L, and LaMaire R (2001) Distributed topology construction of Bluetooth personal area networks. In: Proc. of IEEE INFOCOM. pp. 15771586.

Schurgers C and Srivastava MB (2001) Energy efficient routing in WSNs, In: Proc. of MILCOM Conf.. Vienna. pp. 357-361

Shah RC and Rabaey JM (2002) Energy aware routing for low energy ad hoc sensor networks, In: Proc. of WCNC, pp 350-355

Shakkottai S, Rappaport TS, and Karlsson PC (2003) Cross-layer design for wireless networks. IEEE Communications 41:74-80

Tan G, Miu A, Guttag J, and Balakrishnan H (2001) Forming scatternets from Bluetooth personal area networks. (Technical report MIT-LCS-TR-826 by MIT)

Tan HO and Korpeoglu I (2003) Power efficient data gathering and aggregation in WSNs, ACM SIGMOD Record 32:66-71

Van Hoesel L, Nieberg T., Wu J, and Havinga PJM (2004) Prolonging the lifetime of WSNs by cross-layer interaction. IEEE Wireless Communications 11:78 - 86

Wang Z, Thomas RJ, and Haas Z (2002) Bluenet - a new scatternet formation scheme. In: Proc. of the $35^{\text {th }}$ Hawaii Int. Conf. on System Sciences

Xu Y, Heidemann J, and Estrin D (2001) Geography-informed energy conservation for ad hoc routing. In: Proc. of the ACM/IEEE Int. Conf. on mobile computing and networking. Rome. pp 70-84.

Yao Y and Gehrke J (2002) The Cougar approach to in-network query processing in sensor networks. ACM SIGMOD Record 31:9-18

Ye W, Heidemann J, and Estrin D (2002) An energy efficient MAC protocol for WSNs. In: Proc. of IEEE Infocom Conf.. New York, pp 1567-1576

Yu Y, Govindan R, and Estrin D (2001) Geographical and energy aware routing: a recursive data dissemination protocol for WSNs. (Technical Report UCLA/CSD-TR-010023 by UCLA, 2001.

Zaruba G, Basagni S, and and Chlamtac I (2001) BlueTrees - scatternet formation to enable Bluetooth-based personal area network. In: Proc. of the IEEE Int. Conf. on communications. pp 273-277

Zhang X and Riley GF (2005) Enery aware on-demand scatternet formation and routing for Bluetooth-based WSNs, IEEE Communications 43:126-133

Zhao F, Guibas L (2004) WSNs: an information processing approach. Elsevier-Morgan Kaufmann, Boston

ZigBee Alliance (2004) ZigBee standard specification. Available at: http://www.zigbee.org/en/spec_download/download_request.as 